\title{
Surgical stabilization of shoulder luxation in a pot-bellied pig
}

\author{
Luis M. Rubio-Martínez, DVM, DVsc, PhD, DACvs; Eva Rioja, DVM, DVsc, PhD, DACVA; A. S. Shakespeare, BSc, BVSc, MMedVet
}

\begin{abstract}
Case Description-A 4.6-month-old pot-bellied pig was evaluated because of non-weightbearing lameness (grade 5/5) in the right forelimb of 4 days' duration.
\end{abstract}

Clinical Findings-Clinical and radiographic examination revealed a closed, lateral luxation of the right shoulder joint.

Treatment and Outcome - Initial attempts at closed reduction failed to provide adequate stability of the shoulder joint. Open reduction and internal fixation by placement of 2 lateral tension sutures with a system designed for canine cranial cruciate ligament repair provided adequate joint stability and a successful outcome.

Conclusions and Clinical Relevance-Stabilization of the shoulder joint with lateral tension sutures after open reduction should be considered for management of lateral shoulder luxation in pot-bellied pigs. ( J Am Vet Med Assoc 2013;242:807-811)

A 13.5-kg (29.7-lb) 4.6-month-old male pot-bellied pig was evaluated at the Onderstepoort Veterinary Academic Hospital because of a non-weight-bearing lameness of the right forelimb of 4 days' duration. On evaluation, the pig's vital parameters were within reference limits. A non-weight-bearing lameness was evident in the right forelimb, which was carried in a semiflexed position with a dropped elbow joint. Physical examination of the affected limb revealed no abnormalities distal to and including the right elbow joint. Moderate swelling was present over the right shoulder region, and a painful response was elicited when this area was manipulated.

Radiographs of the right shoulder region were obtaned while the pig was under sedation with azaperone (2 mg/kg [0.9 mg/lb], IM). A closed lateral luxation of the right shoulder joint was diagnosed: the humeral head was located lateral and slightly cranial to the scapula (Figure 1). No other remarkable radiographic findings were present. Flunixin meglumine $(1.1 \mathrm{mg} / \mathrm{kg}$ [0.5 mg/lb], IM, q 24 h) was administered for its antiinflammatory and analgesic properties.

The day after initial evaluation, closed reduction of the luxated joint was attempted. Food was withheld from the pig for 8 hours prior to the procedure. Morphine sulfate $(0.3 \mathrm{mg} / \mathrm{kg}[0.14 \mathrm{mg} / \mathrm{lb}], \mathrm{IM})$ and midazolam hydrochloride $(0.2 \mathrm{mg} / \mathrm{kg}$ [0.09 mg/lb], IM) were administered for premedication. A 24-gauge, 1-inch (25-mm) IV catheter was placed in the auricular vein. After 5 minutes of administration of $100 \%$ oxygen with a tight-fitting mask, general anesthesia was induced

\footnotetext{
From the Departments of Companion Animal Clinical Studies (RubioMartinez, Rioja) and Production Animals Studies (Shakespeare), Faculty of Veterinary Science, University of Pretoria, 0110 Onderstepoort, South Africa. Drs. Rubio-Martínez and Rioja's present address is Equine Division, School of Veterinary Science, University of Liverpool, Leahurst Campus, Neston CH64 7TE, England.

The authors thank Estelle Mayhew for preparing the illustration of the surgical procedure.

Address correspondence to Dr. Rubio-Martinez (luis.rubiomartinez@ hotmail.com).
}

\begin{tabular}{c} 
Abbreviation \\
$\mathrm{CCL} \quad$ Cranial cruciate ligament \\
\hline
\end{tabular}

with propofol $(4.4 \mathrm{mg} / \mathrm{kg}$ [2.0 mg/lb], IV). A 6-mminternal diameter cuffed endotracheal tube was used for orotracheal intubation, and anesthesia was maintained with isoflurane in oxygen administered through a circle breathing circuit. Crystalloid fluids (lactated Ringer's solution) were administered IV at a rate of $10 \mathrm{~mL} / \mathrm{kg} / \mathrm{h}$ $(4.5 \mathrm{~mL} / \mathrm{lb} / \mathrm{h})$ continuously during anesthesia.

To attempt reduction of the luxation, traction was applied to the affected limb and manual pressure was placed on the humeral head while simultaneously abducting and externally rotating the limb. On the basis of findings on repeated radiography, this procedure was unsuccessful. The pig recovered uneventfully from anesthesia. Open reduction and internal fixation were performed 2 days later after obtaining informed consent for surgery from the owner.

Food was withheld from the pig for 8 hours prior to surgery. Trimethoprim-sulphamethoxazole $(20 \mathrm{mg} / \mathrm{kg}$ [9 mg/lb], PO) and meloxicam $(0.5 \mathrm{mg} / \mathrm{kg}[0.23 \mathrm{mg} / \mathrm{lb}]$, PO) were administered before surgery. Administration of flunixin was discontinued and substituted with meloxicam $(0.4 \mathrm{mg} / \mathrm{kg}[0.18 \mathrm{mg} / \mathrm{lb}], \mathrm{PO}, \mathrm{q} 24 \mathrm{~h})$ to reduce the risks of gastrointestinal adverse effects. Premedication, anesthetic induction, and maintenance of general anesthesia were performed as described for the previous attempt at closed reduction of the luxation. The pig's blood pressure, $\mathrm{ECG}, \mathrm{PO}_{2}, \mathrm{PCO}_{2}$, and esophageal temperature were monitored throughout the surgical procedure. During surgery, morphine $(0.3 \mathrm{mg} / \mathrm{kg}$, IV) administration was repeated 2 hours after the preoperative dose as well as a bolus dose of ketamine $(3 \mathrm{mg} / \mathrm{kg}[1.36 \mathrm{mg} / \mathrm{lb}], \mathrm{IV})$ to provide multimodal analgesia. A bolus dose of glycopyrrolate $(0.005 \mathrm{mg} / \mathrm{kg}$ [0.0023 mg/lb], IV) was administered during anesthesia to counteract bradycardia.

During surgery, the patient was positioned in lateral recumbency with the affected limb uppermost. A cranio- 

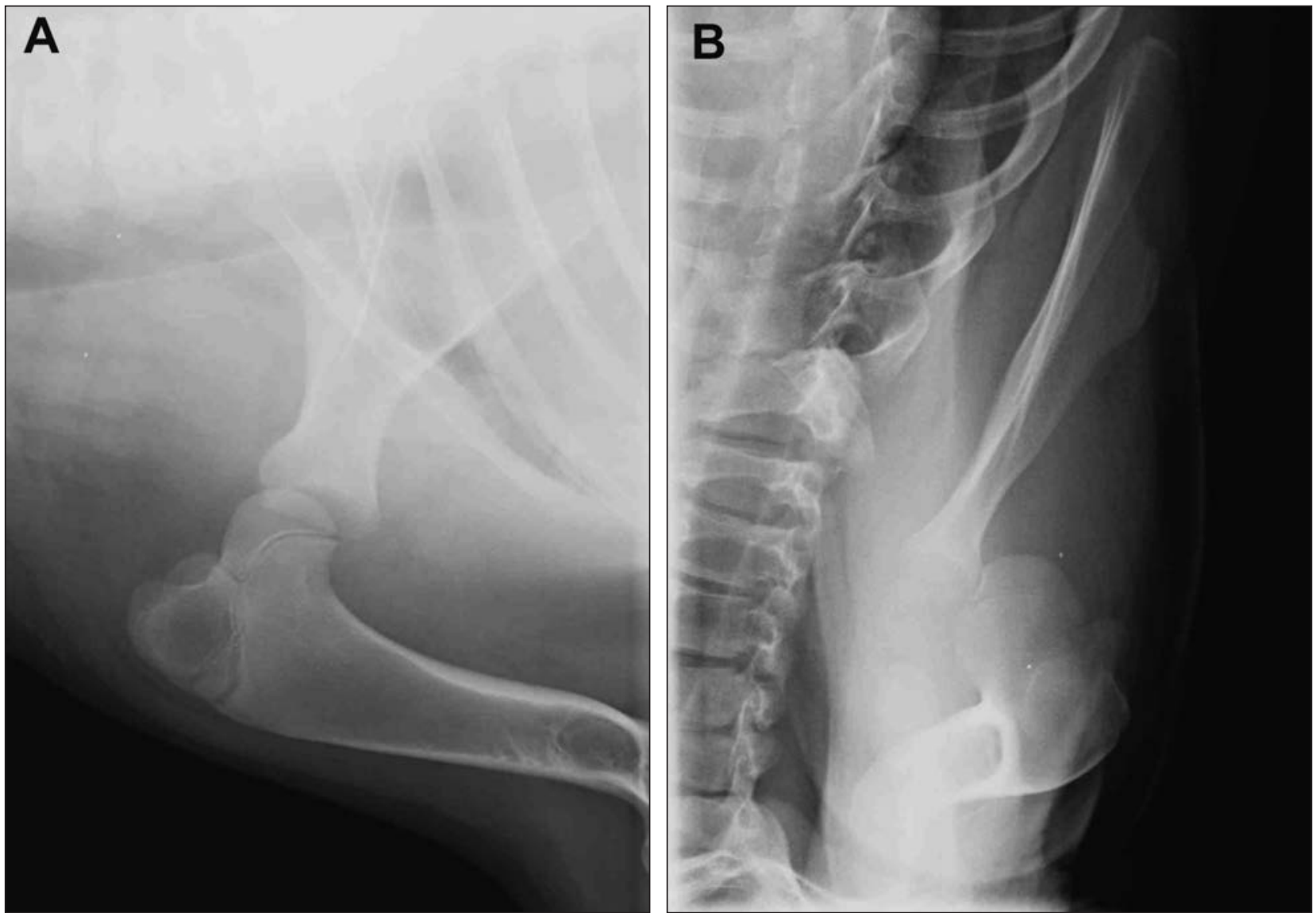

Figure 1-Preoperative radiographic images of the right shoulder region of a 13.5-kg (29.7-lb) 4.6-month-old male pot-bellied pig. Mediolateral (A) and caudocranial (B) views demonstrate a closed lateral luxation of the right shoulder joint. The humeral head is located lateral and slightly cranial to the scapula.
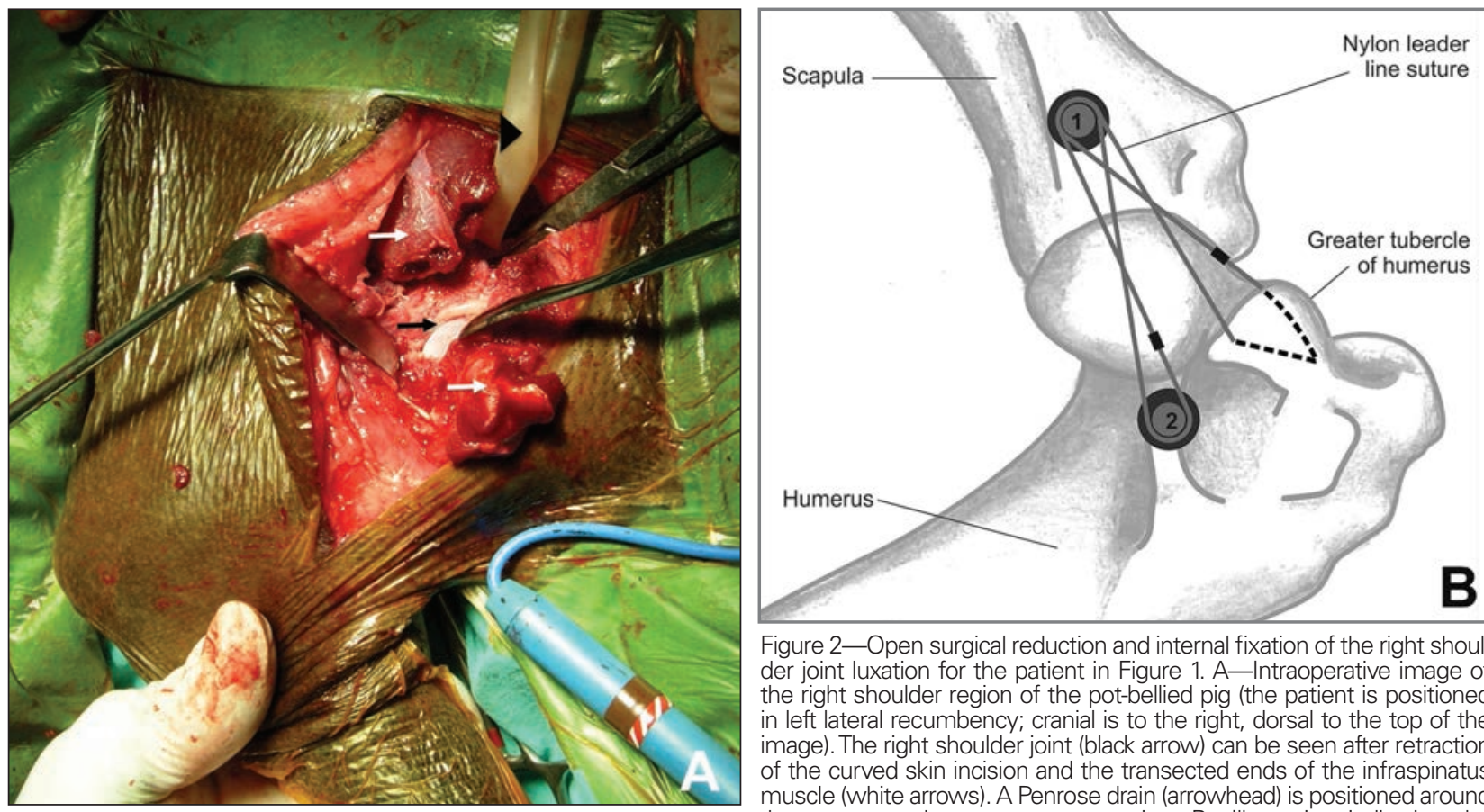

Figure 2-Open surgical reduction and internal fixation of the right shoulder joint luxation for the patient in Figure 1. A-Intraoperative image of the right shoulder region of the pot-bellied pig (the patient is positioned in left lateral recumbency; cranial is to the right, dorsal to the top of the image). The right shoulder joint (black arrow) can be seen after retraction of the curved skin incision and the transected ends of the infraspinatus muscle (white arrows). A Penrose drain (arrowhead) is positioned around the suprascapular nerve as protection. B-lllustration indicating the placement of tension sutures in the right shoulder region (position is similar to panel $\mathrm{A}$; cranial is to the right and dorsal to the top of the image). Two 4.0-mm-diameter cancellous screws equipped with stainless steel washers were placed $(1,2)$; one screw was inserted in the center of the scapular neck (1) and the other in the center of the humeral neck (2). Nylon leader line sutures were placed in a figure-of-eight pattern around these 2 screws. Another similar tension suture was placed with identical nylon suture in a figure-of-eight pattern around the screw placed in the scapular neck and through a 2-mm track (dashed line) drilled through the greater tubercle of the humerus in a caudolateral-craniomedial direction. 


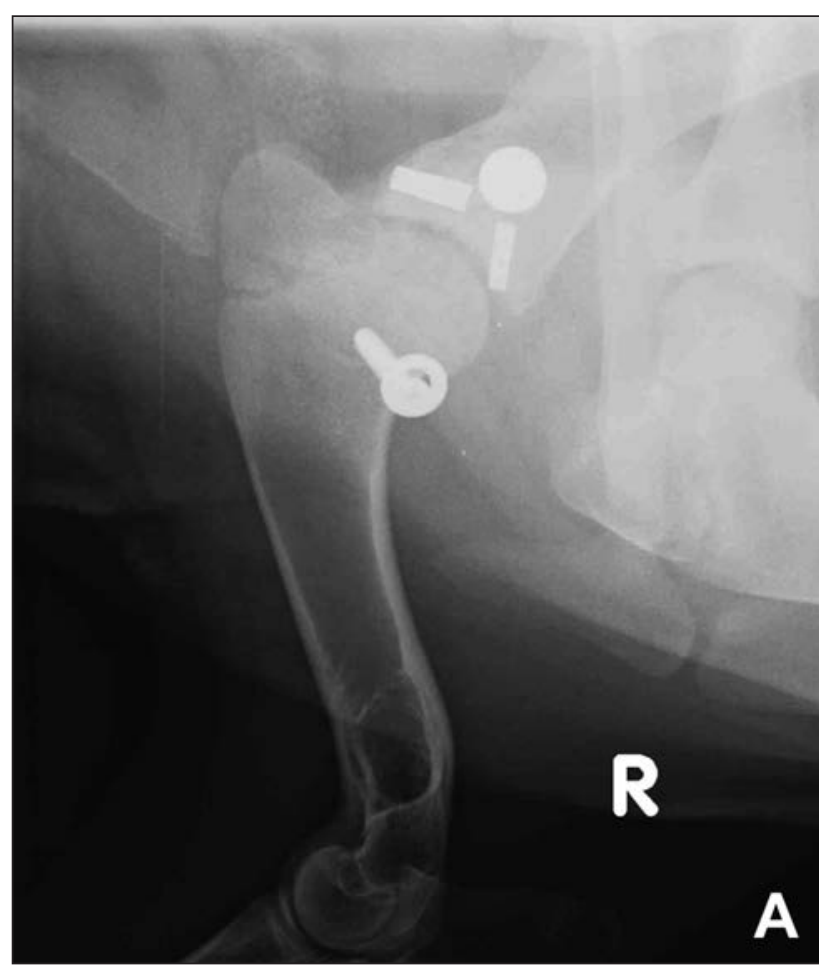

Figure 3-Radiographic images of the right shoulder joint of the patient in Figure 1 obtained 3 months after open reduction and internal fixation with 2 tension sutures. Mediolateral (A) and caudocranial $(B)$ views show that the right shoulder joint is reduced and some periosteal new bone is evident around the scapular neck and the cranial aspect of the humeral head. The screws and metal crimp clamps used for surgical repair are evident on the images.

lateral approach was used as described in other large animal species. ${ }^{1}$ A curved skin incision was made over the right shoulder region. The incision started at the middle of the scapular spine and continued over the lateral surface of the humerus to the midpoint of the humeral shaft at the level of the deltoid tuberosity of the humerus. The deep brachial fascia was incised cranial to the deltoid muscle, with the incision extended caudad to the deltoid tuberosity of the humerus. The intact infraspinatus tendon was transected to obtain adequate exposure of the intact capsule of the shoulder joint.

The joint capsule was incised parallel to the glenoid rim, and the joint was examined (Figure 2). An approximately 1-cm-diameter full-thickness excoriation of the articular cartilage was observed in the center of the humeral head. The joint was reduced by manual traction and adduction with external rotation. Once the joint was aligned, 2 scapulohumeral tension sutures were placed with a nylon suture specifically developed for canine CCL repair. ${ }^{a}$ Two 4.0 -mm-diameter cancellous screws equipped with stainless steel washers were placed; one screw was inserted in the center of the scapular neck and the other in the center of the humeral neck. A nylon leader line suture with an 80-lb breaking strength was placed in a figure-of-eight pattern around these 2 screws. Another similar tension suture was placed with identical nylon suture in a figureof-eight pattern around the screw in the scapular neck and through a 2 -mm track drilled through the greater tubercle of the humerus in a caudolateral-craniomedial

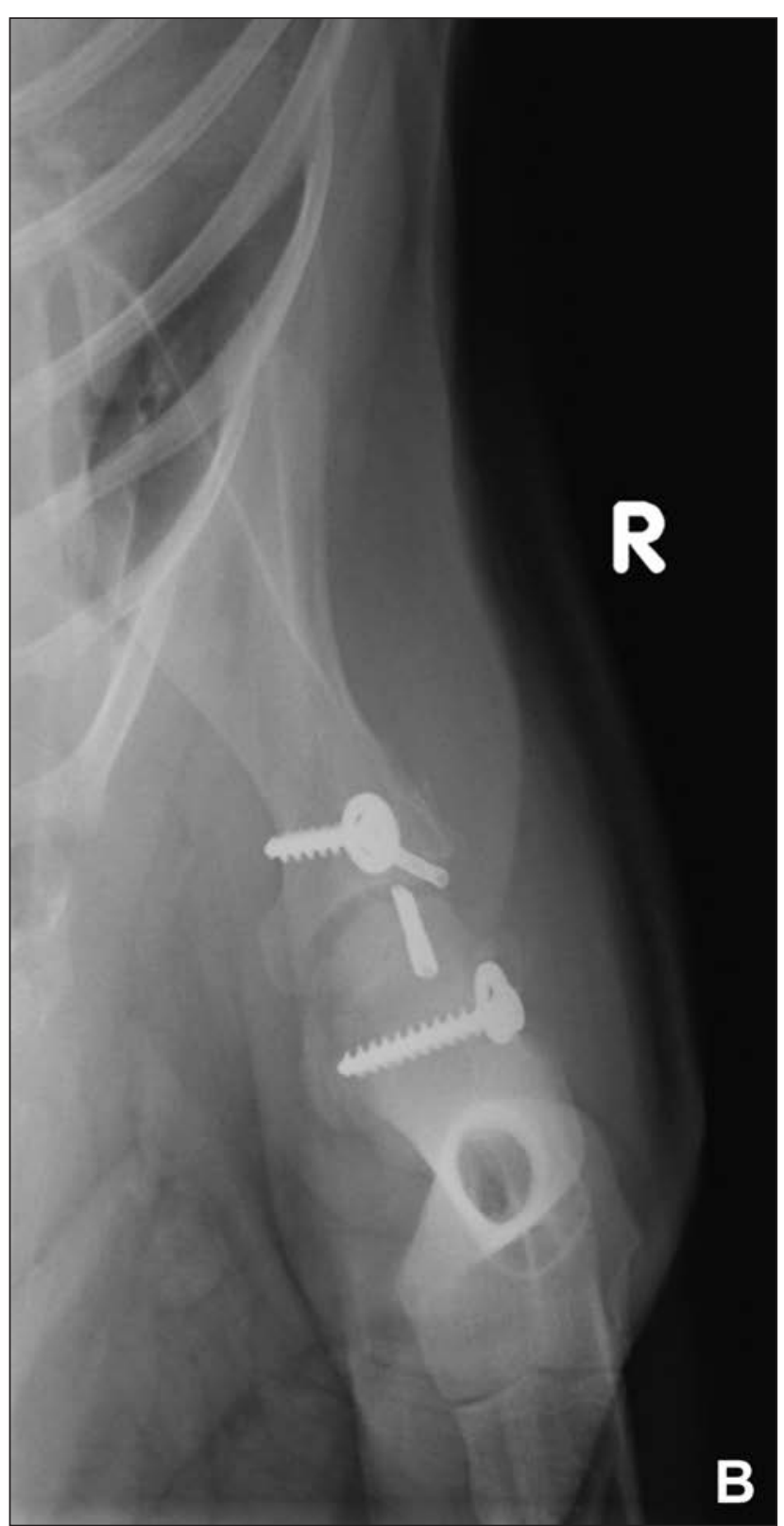

direction (Figure 2). Each tension suture was secured by use of a crimp, with the affected limb held in extension and slightly abducted.

The joint capsule was closed with 2-0 polydioxanone in a simple continuous pattern, and the transected infraspinatus tendon was repaired with 0 polydioxanone in a locking loop pattern. The deep brachial fascia (2-0 polydioxanone), subcutaneous tissues (3-0 polyglecaprone), and skin (2-0 nylon) were closed with a simple continuous suture pattern in separate layers. A sterile adhesive bandage was placed over the surgery site, and the affected limb was immobilized in extension by means of a spica bandage. Postoperative radiographic examination of the right shoulder revealed successful reduction of the luxation. Recovery from anesthesia was uneventful.

After surgery, the pig received meloxicam $(0.4 \mathrm{mg} /$ $\mathrm{kg}$, PO, q 24 h, for 3 days), morphine (0.3 mg/kg, IM, q 
$8 \mathrm{~h}$, for 2 days), vitamin B complex ( $8 \mathrm{~mL}, \mathrm{PO}, \mathrm{q} 24 \mathrm{~h}$, for 2 days), and trimethoprim-sulphamethoxazole (20 $\mathrm{mg} / \mathrm{kg}, \mathrm{PO}$, for 7 days). A tetanus vaccine was also administered. The pig was able to lie down and ambulate with the spica bandage in place. The pig was discharged from the hospital 2 days after surgery.

The owners were instructed to keep the pig in a confined area and monitor the bandage for any problems. The pig was allowed to freely ambulate on the ground floor of the house but not to climb stairs or go outside the house. The owners reported that the pig was active and weight bearing on the affected limb after discharge, although a mild right forelimb lameness was noticeable. The same degree of mild lameness was observed 12 days after surgery when the pig was returned to the hospital for removal of the bandage and skin sutures. After removal of the bandage and sutures, the pig had mild lameness at the walk with weakness of the right forelimb, although full weight bearing was present on the affected limb. The pig was discharged to the owners' care with instructions to restrict exercise. The pig was confined as before for another 2 weeks. After this time, the pig was allowed to go outside but was confined to an area where running was not possible. The amount of exercise was gradually increased over the following weeks until unrestricted exercise was allowed 8 weeks after surgery. The owners reported that the pig had progressed well, and within 2 weeks after discharge, the lameness was not noticeable to them.

Three months after surgery, the pig was returned for a follow-up examination. The surgical site had healed completely without complications. No gait deficits were noticeable when the animal walked. Radiographs were obtained while the pig was under sedation with ketamine $(8 \mathrm{mg} / \mathrm{kg}$ [3.6 mg/lb], IM) and midazolam $(0.5 \mathrm{mg} / \mathrm{kg}, \mathrm{IM})$. The right shoulder joint was still reduced, and some periosteal new bone was evident around the scapular neck and the cranial aspect of the humeral head (Figure 3). Ten months after surgery, the owners reported normal ambulation of the pig.

\section{Discussion}

Luxation of the shoulder joint is an infrequent cause of lameness in large animals, including horses, cattle, goats, Himalayan tahr, and 1 pot-bellied pig. ${ }^{1-10}$ Management of shoulder luxations in these species has been performed by closed reduction and stabilization, $, 3,6,10,11$ open reduction and internal fixation, ${ }^{1,2,6,10}$ and arthrodesis. ${ }^{4,5,7,10}$

In the patient described in the present report, closed reduction of the luxated shoulder joint was unsuccessful, whereas open reduction and application of 2 lateral tension sutures over the shoulder joint provided adequate stability. Closed reduction of acute simple shoulder luxations is associated with a good prognosis in alpacas ${ }^{10}$; however, it was unsuccessful in more chronic cases and other species. ${ }^{4,6,12}$ Recurrence of luxation after successful closed reduction does not seem common in horses, ponies, and female alpacas on the basis of a few studies, ${ }^{3,10,13}$ but is apparently more common in small animals and other large animal species. ${ }^{1,7}$ In our patient and the previously reported case of a pot-bellied pig with shoulder luxation, ${ }^{6}$ closed reduction attempts were unsuccessful. Open reduction and joint stabilization by transposition of the tendon of the biceps brachii muscle, placement of a scapulohumeral tension band, and imbrication of the surrounding musculature provided adequate stability to the affected joint in the previously reported case. ${ }^{6}$ The bicipital tendon transposition technique has also been successfully used in small animals, ${ }^{12}$ nondomestic ruminants, ${ }^{8}$ and an alpaca. ${ }^{9}$ The extracapsular tension suture technique can be readily applied, is less invasive, and has been successfully used in goats, alpacas, and a steer. ${ }^{1,2,10}$ Two tension sutures were used in the pig of the present report for increased rotational stability ${ }^{1,10}$; however, a single tension suture has been used in goats. ${ }^{2}$ We did not observe any damage to the infraspinatus tendon or the joint capsule as commonly found in other large animal species. ${ }^{1,10}$ Transection of the infraspinatus tendon was required for adequate access to the scapulohumeral joint, which was necessary in alpacas but not in a steer. ${ }^{1}$

The CCL suture system is commonly used to provide joint stability in small animals with CCL damage. ${ }^{14}$ The use of the CCL nylon suture system for stabilization of the shoulder joint has not been previously reported in large animal species, but other materials, such as fiberwire $^{1}$ and nylon ribbon, ${ }^{2}$ have been used. A nylon suture with a breaking strength of $80 \mathrm{lb}$ was selected because this caliber is recommended for dogs weighing $15 \mathrm{~kg}(33 \mathrm{lb}){ }^{a}$ This suture system was easy to apply in this case, and the use of the crimp clamps to secure the suture provides increased control and decreases the difficulties during knot tying. ${ }^{15}$

A spica bandage was used to immobilize the limb for 12 days after surgery in the patient described in the present report. The suggested duration of immobilization in small animals after surgical repair or closed reduction of a luxated shoulder joint is 1 to 3 weeks. ${ }^{16}$ Velpeau-type slings have been used in a previous study ${ }^{1}$ after shoulder luxation correction in large animals; however, Velpeau-type slings have not been recommended in instances of lateral shoulder luxations in dogs because of increased lateral rotation of the humerus. ${ }^{12}$ A modified Velpeau sling including a thoracic component was applied after extracapsular stabilization of the shoulder joint in alpacas, with good results. ${ }^{10}$

Degenerative joint disease is a common sequela of shoulder luxation and was radiographically evident after open reduction and correction of shoulder luxation via tendon transposition in a pot-bellied pig. ${ }^{6}$ The same was also evident in this case; however, neither of these animals was lame on follow-up examination.

The cause of shoulder luxations is commonly traumatic, but congenital shoulder luxations have also been reported in small animals and alpacas. ${ }^{10,17}$ Dysplasia of the shoulder joint has also been involved in shoulder luxations in ponies. ${ }^{7,18,19}$ Sexually intact male alpacas seem to be more affected by shoulder luxations than females, which may be a result of the more active and more aggressive behavior of sexually intact males. ${ }^{1,10}$

The present report describes the successful repair of a shoulder luxation in a small pot-bellied pig with the use of lateral tension sutures. On the basis of the 
limited literature ${ }^{5}$ and this case, open reduction and internal stabilization appear to offer better results than closed reduction of shoulder luxations in pot-bellied pigs. The nylon suture system developed for canine CCL repair can be successfully applied as tension sutures to stabilize shoulder luxations in pot-bellied pigs.

a. CCL Lateral Suture System, Joergensen Laboratories Inc, Loveland, Colo.

\section{References}

1. Watts AE, Fortier LA, Nixon AJ. A technique for internal fixation of scapulohumeral luxation using scapulohumeral tension sutures in three alpacas and one miniature steer. Vet Surg 2008;37:161-165.

2. Purohit NR, Choudhary RJ, Chouhan DS, et al. Surgical repair of scapulohumeral luxation in goats. Mod Vet Pract 1985;66:758-759.

3. Madison JB, Young D, Richardson D. Repair of shoulder luxation in a horse. J Am Vet Med Assoc 1991;198:455-456.

4. MacDonald DG, Bailey JV, Fowler JD. Arthrodesis of the scapulohumeral joint in a horse. Can Vet J 1995:36:312-315.

5. Arighi M, Miller CR, Pennock PW. Arthrodesis of the scapulohumeral joint in a miniature horse. J Am Vet Med Assoc 1987;191:713-714.

6. Semevolos SA, Nixon AJ, Goodrich LR, et al. Shoulder joint luxation in large animals: 14 cases (1976-1997). J Am Vet Med Assoc 1998;213:1608-1611.

7. Semevolos SA, Watkins JP, Auer JA. Scapulohumeral arthrodesis in miniature horses. Vet Surg 2003;32:416-420.

8. Howard LL, Richardson GL. Transposition of the biceps tendon to reduce lateral scapulohumeral luxation in three species of nondomestic ruminant. J Zoo Wildl Med 2005;36:290-294.

9. Vandeweerd JM, Clegg P, Wawa E. Treatment of recurrent luxation of the shoulder in an alpaca. Vet Rec 2007;160:304-306.

10. Rousseau M, Anderson DE, Miesner MD, et al. Scapulohumeral joint luxation in alpacas: 10 cases (2003-2009). J Am Vet Med Assoc 2010;237:1186-1192.

11. Hardy J, Marohn MA. What is your diagnosis? Scapulohumeral luxation. J Am Vet Med Assoc 1989;195:1773-1774.

12. Piermattei DL, Flo GL. In: Piermattei DL, Flo GL, eds. Brinker, Piermattei and Flo's handbook of small animal orthopedics and fracture repair. Philadelphia: WB Saunders Co, 1997;231-238.

13. Wilson RG, Reynolds WT. Scapulohumeral luxation with treatment by closed reduction in a horse. Aust Vet J 1984;61:300301.

14. Lampman TJ, Lund EM, Lipowitz AJ. Cranial cruciate disease: current status of diagnosis, surgery, and risk for disease. Vet Comp Orthop Traumatol 2003;16:122-126.

15. Scherzer S, Hainisch EK. Evaluation of a canine cranial cruciate ligament repair system for use in equine laryngoplasty. Vet Surg 2005;34:548-553.

16. Davidson JR, Kerwin SC, Millis DL. Rehabilitation for the orthopedic patient. Vet Clin North Am Small Anim Pract 2005;35:1357-1388

17. Vaughan LC, Jones DG. Congenital dislocation of the shoulder joint in the dog. J Small Anim Pract 1969;10:1-3.

18. Boswell JC, Schramme MC, Wilson AM. Radiological study to evaluate suspected scapulohumeral joint dysplasia in Shetland ponies. Equine Vet J 1999;316:510-514.

19. Clegg PD, Dyson SJ, Summerhays GE. Scapulohumeral osteoarthritis in 20 Shetland ponies, miniature horses and Falabella ponies. Vet Rec 2001;148:175-179. 